GT2017-63079

\title{
HIGH-FIDELITY SIMULATIONS OF A LINEAR HPT VANE CASCADE SUBJECT TO VARYING INLET TURBULENCE
}

\author{
Richard Pichler* and Richard D. Sandberg \\ Department of Mechanical Engineering \\ University of Melbourne, VIC 3010, Australia \\ Email: richard.pichler@unimelb.edu.au
}

\author{
Gregory Laskowski \\ General Electric Aviation \\ Lynn, MA, USA
}

\author{
Vittorio Michelassi \\ General Electric Oil and Gas \\ Florence, Italy
}

\begin{abstract}
The effect of inflow turbulence intensity and turbulence length scales have been studied for a linear high-pressure turbine vane cascade at $R e_{i s}=590,000$ and $M_{i s}=0.93$, using highly resolved compressible large-eddy simulations employing the WALE turbulence model. The turbulence intensity was varied between $6 \%$ and $20 \%$ while values of the turbulence length scales were prescribed between $5 \%$ and $20 \%$ of axial chord. The analysis focused on characterizing the inlet turbulence and quantifying the effect of the inlet turbulence variations on the vane boundary layers, in particular on the heat flux to the blade. The transition location on the suction side of the vane was found to be highly sensitive to both turbulence intensity and length scale, with the case with turbulence intensity $20 \%$ and $20 \%$ length scale showing by far the earliest onset of transition and much higher levels of heat flux over the entire vane. It was also found that the transition process was highly intermittent and local, with spanwise parts of the suction side surface of the vane remaining laminar all the way to the trailing edge even for high turbulence intensity cases.
\end{abstract}

\section{NOMENCLATURE}

Re, Ma Reynolds and Mach number

$C, C_{a x} \quad$ true and axial blade chord length

$c_{p} \quad$ blade pressure coefficient

$q, q_{n} \quad$ heat flux and normalized heat flux

$k \quad$ turbulence kinetic energy

$\rho, u_{i}, p \quad$ density and velocity component and pressure

$\omega$

$x, y, z$ wake loss coefficient

axial, pitchwise and spanwise coordinates

\footnotetext{
${ }^{*}$ Address all correspondence to this author.
}

\section{Subscripts and Superscripts \\ 1,2 inlet and outlet plane \\ is isentropic exit values \\ $\infty$ reference value \\ $+\quad$ nondimensional wall unit \\ $t \quad$ stagnation value \\ rms root mean square}

\section{INTRODUCTION}

The operating conditions of the high pressure turbine (HPT) in current engine designs are such that they require cooling. While it is necessary to limit the HPT blade wall temperature to maintain turbine life it reduces the thermal efficiency. In order to design effective and efficient cooling schemes, a detailed understanding of the gas temperature and wall heat transfer in the absence of cooling is required. Because both of these are strongly influenced by turbulence, shocks and wake mixing, studies conducted in the absence of cooling will enable designers to understand how much cooling air is actually required to achieve desired metal temperatures. In addition, understanding the uncooled performance helps to quantify the impact of cooling on HPT performance. For example, Leach [1] measured the impact of coolant flow on aerodynamic efficiency of an HPT stator, quantifying that cooling resulted in an additional 3.5\% total pressure loss drop at 50\% span compared to a non-cooled stator.

A number of studies have demonstrated the importance of free stream turbulence on the heat flux. Art and Rouvroit [2] conducted a detailed study of an uncooled, transonic linear vane cascade at 
exit Reynolds numbers $0.5 \times 10^{6}<R e<2 \times 10^{6}$, exit Mach numbers between 0.7-1.2 and turbulence intensities from $1 \%$ to $6 \%$. At an exit Reynolds number of 590,000 variation of inlet turbulence from $1 \%$ to $6 \%$ resulted in a shift of the transition location on the suction side of $10 \%$ surface length. Variation of inlet turbulence intensity was achieved by changing the upstream position of the bar grid used to generate turbulence. This is expected to also vary the resulting turbulence length scale of the inlet condition, however, this effect was not discussed. In an effort to get closer to engine-like turbulence intensities, Nix [3] showed experimentally that increasing Tu from $2 \%$ to $16 \%$ increased the pressure side heat transfer by $50 \%$ while having little impact on the suction side surface. This suggests that the strong acceleration of the flow on the suction side reduced the local turbulence intensity to amplitudes sufficiently low so that the transition behavior was not affected. The impact of turbulence levels on the flow physics has been reported in the literature and has received renewed attention $[4,5]$.

As discussed above transition plays a key role for design critical decisions. Coull and Hodson [6] discuss different "routes to transition" they observed in low pressure turbine-like flat plate experiments subject to different incoming perturbations of discrete and homogenous nature. While in the case of discrete disturbances complex unsteady interactions occur, uniform disturbances are mostly discussed in terms of streaks and turbulent spots as already discussed by Kleabanoff [7]. The boundary layer subject to free stream turbulence shows local high fluctuations in the streamwise velocity component that in general have a longer extension in the streamwise than in the transversal direction. Eventually these so called streaks lift up and start breaking down into turbulence (for more details see also Durbin and Wu [8]). Westin et al. [9] showed that apart from turbulence intensity (TI) also anisotropy, length scales (LS) and energy spectra have an influence and should be included in the discussion. A systematic study by Brandt et al. [10] discussed the influence of the LS and concluded that at the same TI a larger LS leads to smaller fluctuations close to the leading edge. However, the structures introduced by turbulence of larger LS grow faster and lead to earlier transition further downstream. As discussed above, apart from the state of the turbulence it has been shown that the development of the boundary layer can affect the transition location significantly [11]. In particular the acceleration inside a boundary layer can suppress the growth of disturbances or lead to their decay. While all of the mechanisms discussed so far have been found to influence transition in an HPT, the combined effect of all of them has not yet been thoroughly investigated.

In this work we conduct a systematic study of the effect of turbulence length scale and intensity on the transition mechanisms close to realistic operating conditions on a realistic HPT vane geometry using highly resolved large eddy simulations (LES). The case is that experimentally investigated by Arts and Rouvroit [2] and previously simulated using DNS with the same high-fidelity code by Wheeler et al. [12]. In Pichler et al. [13] an LES setup was presented that showed no differences to the DNS by Wheeler et al. [12] in the transition location and this setup is used in this work as well. The results are compared against experiments where available and the trends of transition location and evolution with respect to varying inlet turbulence parameters are discussed.

\section{NUMERICAL METHOD}

HiPSTAR is a University of Melbourne in-house multi-block structured compressible Navier-Stokes solver purposely developed and optimized to exploit the latest massively parallel highperformance computing systems. Here, only a short summary of the key features of the numerical method are given. For a comprehensive presentation of the code and its performance, see Sandberg et al. [14] and Michelassi et al. [15]. In the current study, the spatial derivatives in the axial/pitchwise plane are computed using fourth-order accurate explicit central finite-difference schemes with energy-preserving fourth- order accurate boundary schemes [16]. The discretization in the homogeneous spanwise direction is accomplished with a spectral approach, using the FFTW library. For the advancement in time, an explicit fourth-order Runge-Kutta method is used. The code has been thoroughly tested for accuracy on a number of canonical test cases, transonic tip flows [17] and against T106A low pressure turbine (LPT) linear cascade measurements [14], [15]. Initially, HiPSTAR was used to conduct DNS of the LPT at different Reynolds numbers and reduced frequencies, thus fully resolving the flow. More recently, LES models have been implemented into the code and the suitability and accuracy of the standard WALE model, described in [18], was demonstrated in another LPT study that investigated the concerted action of reduced frequency and flow coefficient on unsteady loss generation [19]. An explicit filter described in [20] is applied at the end of each Runge-Kutta step and the filter weight is set to 0.15 . For the linear cascade investigation of the HPT vane presented in figure 1, the same grid topology as outlined in [14] was used, comprising four blocks for the O-type grid around the vane to ensure optimal grid quality in the vicinity of the wall, and five blocks for the H-type grid allowing for pitchwise periodicity. In order to ensure high-quality solutions at the block interfaces despite metric discontinuities, all blocks were connected to each other using characteristic interface conditions. At the wall a no-slip isothermal boundary condition is used. The non-reflective zonal characteristic boundary condition described in [21] has been used at the outlet and Riemann invariant boundary conditions have been used at the inlet. In the spanwise homogenous direction a Fourier method is applied implying periodic boundary conditions and an equidistant grid representing the midspan section of the vane.

In the following data are plotted and discussed along the surface length $s$ of the vane, as presented in in Figure 1. It is defined such that it $s=0$ at the LE, negative values indicate the pressure side and it takes positive values on the suction side. The strong flow acceleration on the blade occurs up to $s=1.3$ on the suction side. In this region it is expected that the acceleration of the flow suppresses structures that might lead to transition.

The wall normal spacing is below $y^{+}=2$ except for in the leading and trailing edge regions, and the spacing in the spanwise and the streamwise directions is less than 20 and 50 wall units over most of the vane, respectively. A grid convergence study has not been conducted due to the similarity of the current work with our previous work [13] where good agreement with the DNS [12] was already shown. In the blade-to-blade plane, the number of grid points is kept the same as in [13]. In the spanwise direction the number of grid points is set to result in the same spanwise resolution as in [13]. However, in the current study the spanwise domain size was increased significantly to account for the increase of the turbulence length scales (LS) investigated. As will be shown later, it was found that a spanwise domain size of four times the turbulence length scale was required to achieve grid independent results. 


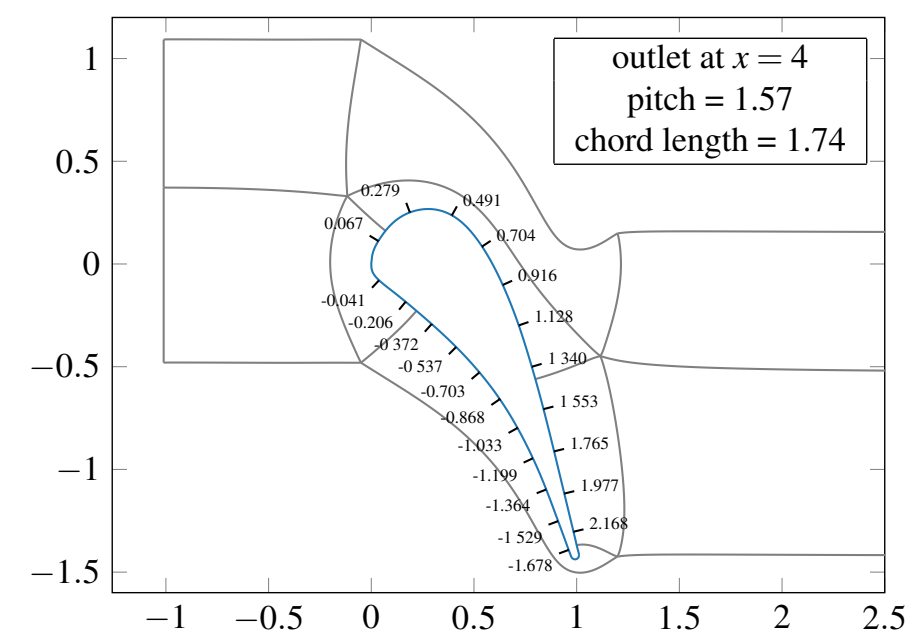

FIGURE 1: DOMAIN SETUP AN ILLUSTRATION OF THE RELATION OF SURFACE LENTGH AROUND THE BLADE SURFACE LENGTH AROUND BLADE, $s$, FOR BOTH PRESSURE AND SUCTION SIDE, WHERE THE LEADING EDGE IS AT $s=0$.

For the case with $5 \% C_{a x}$ turbulent LS the spanwise extent was set to $30 \% C_{a x}$ to take into account the domain size criteria imposed by the wake breakdown mechanisms, requiring $24 \% C_{a x}$. Accordingly, the number of points in the spanwise direction (see table 1) were set to 386 for the $5 \% C_{a x}$ inlet turbulence LS case and 1026 for the $20 \% C_{a x}$ turbulence LS case, resulting in a total of approximately 250 Million and 800 Million grid points, respectively.

\section{TEST CASE}

HPT vane geometry and setup have been selected based on Arts and Rouvroit [2] and the results published in there are used for comparison. The experiments were conducted at an inlet angle of attack of 0 degrees (wrt. the axis) for various cases at different Reynolds and Mach number conditions, representative of engine operating conditions. This study focuses on the case at isentropic exit Reynolds number 590,000 and isentropic exit Mach number 0.93 with a TI of $6.0 \% u_{\infty}$ at $x / c_{a x}=-1.49$. TI in the experiment is defined as

$$
T I=\frac{\sqrt{2 / 3 k}}{u_{\infty}},
$$

where $k$ is the turbulence kinetic energy and $u_{\infty}$ is the inlet velocity. Given only one velocity component was measured experimentally, the turbulent kinetic energy is defined as

$$
k_{\text {exp }}=\frac{3}{2}\left(u_{\text {exp }, r m s}\right)^{2}
$$

where $u_{\text {exp,rms }}$ is the streamwise fluctuating velocity component. For this simulations all quantities presented in this paper are nondimensionalized, with lengths and distances divided by the axial chord $\left(C_{a x}\right)$, velocity and density are normalized by reference inlet conditions and the reference temperature is $300 \mathrm{~K}$. Based on the non-dimensionalization of length and velocity the reference time is $t_{\infty}=C_{a x} / u_{i n}$. Hence one time unit represents the time it takes a
TABLE 1: TEST CASE MATRIX, $z_{L}$ DENOTING SPANWISE DOMAIN SIZE AND $n z$ IS THE NUMBER OF SPANWISE POINTS

\begin{tabular}{c|c|c|c|c} 
case & TI $\left[\% u_{\infty}\right]$ & LS $\left[\% C_{a x}\right]$ & $z_{L}\left[\% C_{a x}\right]$ & $n z$ \\
\hline TI06 LS05 & 6 & 5 & 30 & 386 \\
TI20 LS05 & 20 & 5 & 30 & 386 \\
TI06 LS20 & 6 & 20 & 80 & 1026 \\
TI20 LS20 & 20 & 20 & 80 & 1026
\end{tabular}

particle to move a distance of an axial chord at inlet velocity which in the following is referred to as one flow-through time.

Since the target of this study is to evaluate the influence of transition by varying LS and TI of the incoming turbulence, four cases as summarized in table 1 have been conducted. Two cases have been conducted at TI of $6 \% u_{\infty}$, to allow for comparison with one of the cases experimentally investigated. To obtain more engine-like conditions, the second set of simulations is conducted with TI set to $20 \% u_{\infty}$. In the original report of the experiment no length scale was provided for the incoming turbulence. However, the turbulence LS of the facility is estimated to be of the order of 20 to 30 percent of the axial chord length [22] where larger length scales were present when the grid was further upstream and the turbulence intensity was smaller. Considering homogenous isotropic turbulence based on the decay, Jee et al. [23] estimate the LS to be $40 \%$ percent axial chord length for the same facility. Further, they identify the high frequency peak in the turbulent spectra to be at the same frequency as the vortex shedding of the cylinders used in the turbulence grid at the given flow conditions. This could suggest that the turbulence generated by the grid has not fully broken down to an isotropic state by the time it has reached the measurement position. Length scales as present in the experiment or real engines are difficult to achieve in highly resolved simulations since they require large simulation domains. The largest length scale that can be simulated with the domain used in this study is approximately $20 \% C_{a x}$ as will be shown later and this LS is used for two cases. The other two cases are conducted at a LS of $5 \% C_{a x}$. It should be noted that the values stated here are those prescribed in the inlet turbulence generation method discussed in the next section and will change throughout the domain such that it is not straight forward to separate the influence of varying TI from the influence of varying $\mathrm{LS}$.

\section{INLET TURBULENCE}

Inlet turbulence generation is a topic on its own. While various methods are available, including synthetic methods, recycling methods or simulating an upstream turbulence grid, in this work we have chosen to use a method based on digital filtering, originally proposed by Klein et al. [24], modified for reduced cost by Xie and Castro [25] and adapted for compressible flows by Touber and Sandham [26]. This method generates a two dimensional field of random perturbations at each time step and a digital filter is used to achieve turbulence-like statistics of the signal with a particular focus on the larger scales, while the smaller scales have to develop inside the domain. The filtered fields are correlated in time such that the time coherence of turbulence is achieved. However, the cost of this method scales quadratically with the ratio of the LS 
to the grid spacing which for the ratios required in this work becomes prohibitive. Therefore, in this work turbulence is generated on a separate (coarser) grid and then interpolated onto the LES grid by means of bilinear interpolation. Considering that the inlet turbulence field is synthetically generated and has to develop inside the domain, the interpolation order is not considered critical. In a precursor study it was concluded that the grid for turbulence generation requires 17 points per turbulent LS. Using this approach, the cost of turbulence generation is below $3 \%$ of the total simulation cost.

In another precursor study considering only the inlet region, the parameters required to obtain the desired inlet turbulence were identified. For this study a pitchwise and spanwise periodic domain was considered where the domain size in both directions was varied between 3, 4 and 8 times the turbulence LS. The length of the domain was set to $2 C_{a x}$ and the required resolution as well as development length were tested. In terms of the pitchwise domain size the results are almost identical for domain size 4 or 8 times the turbulence LS, and only very small differences were observed for the domain with 3 times the turbulence LS. For a case with a prescribed TI of 5\% and a LS of $2 \%$ axial chord, doubling the resolution resulted in TI changing from $4.57 \%$ to $4.64 \%$. However, the difference is almost entirely introduced at the inlet boundary and is due to the difficulty of combining nonreflective boundary conditions with prescribing velocity fluctuations. The actual decay inside the domain is similar for both cases. For larger LSs the discrepancies are smaller which is related to the smaller gradients at the inlet boundaries allowing for more reproducible turbulence at the first grid cell. In the initial part of the domain the synthetic inlet turbulence has to develop and it has been found that after about 4 to 5 length scales the inlet turbulence shows decay rates and spectra as expected from homogenous isotropic turbulence.

As discussed by Westin et al. [9] the actual characteristics of turbulence can influence the transition behavior and should be quantified for discussions of transition. Therefore in the following, the relevant turbulence properties are presented and discussed. All results reported are taken from our compressible simulations and therefore also contain contributions of the upstream traveling acoustic waves generated by the interaction of the flow with the trailing edge. These acoustic contributions, however, are typically less than $0.5 \%$ amplitude level [14]. The decay of TI is presented in Figure 2 for all three velocity fluctuation components for each of the four cases considered. Line colors are used to distinguish the different cases and line styles are used to indicate the three velocity components. Westin discussed that the wall normal velocity fluctuations have a more pronounced effect on transition, and hence it is of interest to assess the level of anisotropy of the inlet turbulence. While the two transversal components show similar intensity levels, the streamwise component is significantly larger, thus the inlet turbulence is not isotropic. This is related to the difficulty of feeding in the streamwise component using a nonreflective Riemann invariant boundary condition. The level of anisotropy is larger for the cases with larger LS. However, it is unlikely for the inlet turbulence in the experimental facility or in a real HPT environment to be fully isotropic and hence the authors believe the current simulations to be meaningful.

The cases with smaller LS develop faster from the their synthetic nature to developed turbulence (determined by identifying from when they exhibit decay) and at approximately $\mathrm{x}=-0.75$ exhibit a decay behaviour as expected from the precursor study. For the larger LS it takes up to $\mathrm{x}=-0.1$ until all components have de-

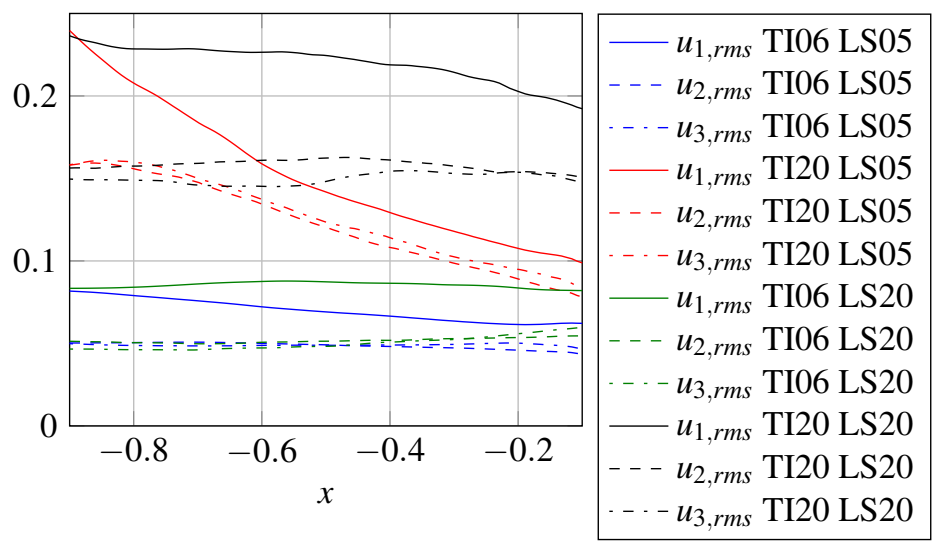

FIGURE 2: INLET TURBULENCE DECAY BETWEEN THE INLET AND THE LEADING EDGE (AT $x=0.0$ ) FOR THE DIFFERENT CASES.

veloped a decay behavior. For the larger length scale cases the inlet turbulence still shows slight wiggles which is due to insufficient statistical convergence in time. Currently 6 flow through times based on inlet velocity and axial chord (more than 30 flow through times based on isentropic exit velocity) are available for the larger LS simulations, which is not enough to fully converge the statistics in the inlet region. However, it is believed that the trends seen are reliable and the absolute values will not change significantly. For the $5 \% C_{a x}$ length scale simulations the statistics are fully converged.

From theory it is known that a larger LS leads to a smaller decay rate. Also the decay rate is greater for larger amplitudes of TI. Both phenomena can be seen in Figure 2. The highest turbulent intensities combined with the smaller length scale exhibits the highest decay rates (TI20 LS05). A larger LS leads to an overall smaller decay rate (TI20 LS20). For the small TI cases the decay rate of the fluctuations of the streamwise velocity component again is smaller than for the large LS cases and the domain length is not sufficiently large to make a definite statement of the decay behavior of the transversal components. However, in the precursor study, where a larger streamwise domain with constant pressure was considered, the decay rates for those fluctuating components were indeed smaller for the large LS than for the small LS, even though the differences were not as pronounced as in the larger TI cases.

The inlet turbulence length scales in the transversal direction at $x=-0.3$ have been confirmed to agree with the prescribed inlet values by computing the autocorrelations in planes normal to the inlet flow direction. The streamwise length scales were also investigated using autocorrelations of the time series at $x=-0.3$. However, because full statistical convergence was not achieved, the data from the precursor simulations of the inflow domain only, for which the same inlet parameters were used, were scrutinized, which showed the resulting LS at $x=-0.3$ to be approximately $5 \%$ to $10 \%$ larger than the prescribed one.

Spectra of the inlet turbulence at an axial position of $x=-0.3$ are presented in Figure 3 for the pitchwise velocity component where the frequency is normalized as

$$
\mathrm{ST}=\frac{f C_{a x}}{u_{\text {in }}},
$$




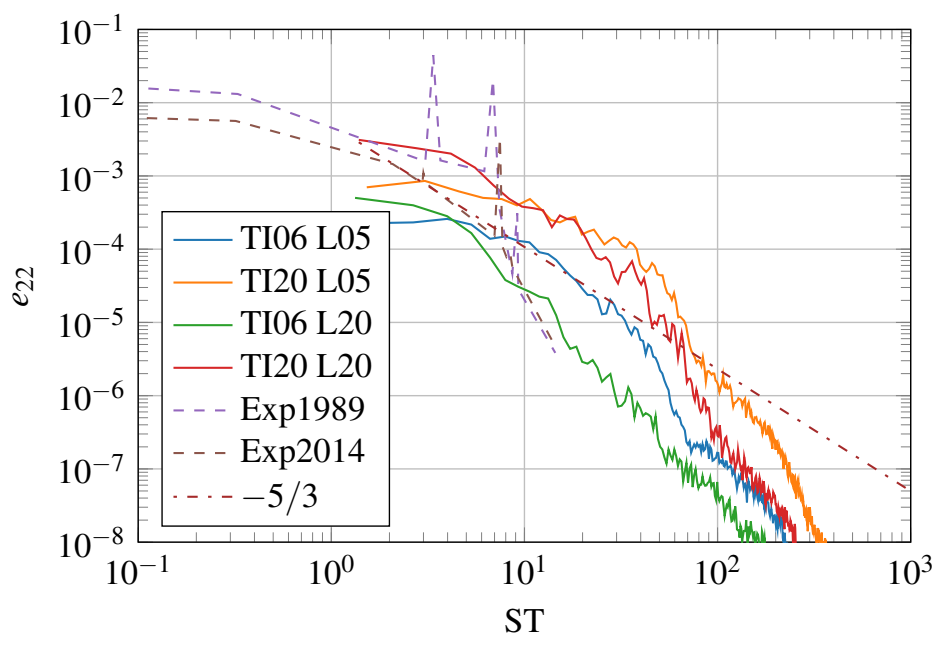

FIGURE 3: INLET TURBULENCE SPECTRA AT $x=-0.3$ FOR ALL CASES WITH EXPERIMINETS IN 1989 [2] AND IN 2014 [22].

and $f, C_{a x}$ and $u_{\text {in }}$ are the frequency, the axial chord and the inlet velocity, respectively. Given that the integral over all frequencies is the energy content, more energy at larger scales, or lower frequencies when invoking Taylor's hypothesis, requires that there is less energy at smaller scales for a fixed TI. Hence, as the spectra in Figure 3 show, for cases with larger integral LS there is more energy in low frequencies and the smaller length scale cases have a higher energy content in the higher frequencies. Additionally, spectra are included from the experiment [2] for a grid position that results in turbulence intensity of 4\% (EXP1989) and from a similar study (EXP2014) conducted more recently by [22], where the grid position is such that the turbulence intensity is $6 \%$ (similar to the one in this study). However, it is not clear how those spectra where normalized so the actual magnitude difference cannot be examined. For comparison purposes, the magnitudes in Exp2014 are multiplied with $10^{-7}$ to be in the same range as the original experiment and the current study.

The experimental spectra show two distinct peaks in addition to a broad turbulence spectrum. Jee et al. [23] realized that the peak at $\mathrm{ST} \approx 3$ is close to the vortex shedding frequency of the bars used in the turbulence grid. In Exp2014 this peak has a smaller amplitude and as can be seen in [22] it is more apparent for cases at lower turbulence intensities. Since those are characterized by a larger distance from the grid, it is conceivable that these structures are breaking down at a smaller rate when passing through the vane which might have an influence on transition. The second peak that is clearly visible in both cases is located at $\mathrm{ST} \approx 7$ and it has been suggested that it is related to acoustic signals. As discussed by Fontaneto [22] this peak is even present when the turbulence grid is removed from the experimental setup and therefore has to be due to another yet unidentified source. However, its magnitude is significant and it is possible that it has an influence on transition as well. While it would be possible to include the turbulence grid in the simulations, which would impose substantially higher cost, the second peak would most likely still not be accounted for. Therefore, the aim of this study is not to try to iterate the setup until perfect agreement is obtained with an experiment for which not all details are known or fully reported, but to use the reference experiment and a previously conducted DNS of the same case to build

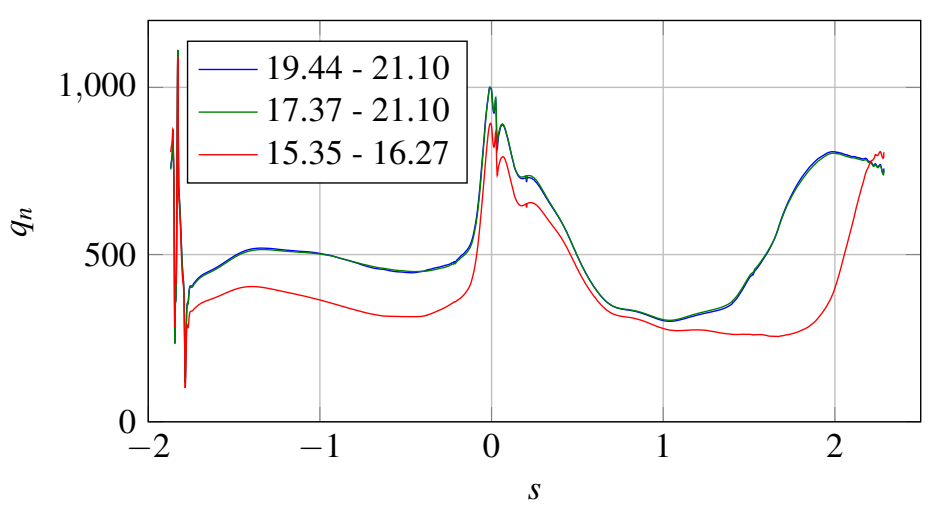

FIGURE 4: WALL HEAT FLUX FOR CASE TI20 L20 ALONG THE BLADE SURFACE $S$, WHERE 0 IS THE LEADING EDGE AND NEGATIVE VALUES INDICATE THE PRESSURE SIDE AND POSITIVE VALUES ARE ON THE SUCTION SIDE. THE FIRST INTERVAL FROM 15.35 TO 16.27 SHOWS RESULTS WHERE THE INFLOW TURBULENCE HAS NOT YET ARRIVED AT THE LEADING EDGE OF THE BLADE. FOR THE OTHER TWO INTERVALS THE INLET TURBULENCE HAS CONVECTED THROUGH THE ENTIRE COMPUTATIONAL DOMAIN.

trust in the ability of the LES setup to reproduce the correct physics and then perform a series of controlled numerical experiments to study the effect of variations in the turbulent inflow characteristics.

While the turbulence, as stated earlier, is not homogenous and isotropic, its spectral shape does resemble real turbulence reasonably well. More importantly, the pronounced differences in spectral shape and amplitude for the four cases with varying LSs and TIs provide an ideal set of cases to study the influence of various inflow conditions on transition. As alluded to earlier, for the large LS cases, the inlet turbulence is not fully statistically converged due to the very high computational cost of this largest case, and therefore a thorough investigation of the statistical convergence of transition phenomena on the blade was performed first for the large LS cases. The heat flux was chosen as a suitable metric for elucidating the onset of laminar-turbulent transition, defined as a normalized heat flux $\left(q_{n}\right)$ [12]

$$
q_{n}=\frac{C_{a x} q}{k_{w}\left(T_{t, i n}-T_{w}\right)}
$$

This is similar to a Nusselt number except that it is affected by both changes in heat transfer coefficient and wall temperature $\left(T_{w}\right)$; the latter will vary significantly at high Mach numbers. Turbulent boundary layers in general cause higher heat flux to the surface with respect to laminar ones. Therefore, a sudden rise in heat flux can indicate the transition. The wall heat flux for case TI20 L20 is plotted in Figure 4 where the leading edge is at $s=0$ with the suction side extending to positive values and the pressure side extending to negative values of $s$. Three different time intervals of the same simulation are presented, where the simulation is initialized using the simulation TI06 LS05 as initial condition at time 15.35. Since it takes about one time unit for the turbulence to arrive at the leading edge, in the interval 15.35 to 16.27 the blade has not yet experienced perturbations with the large LSs and TIs and this interval essentially shows the wall heat flux for the case TI06 LS05. 


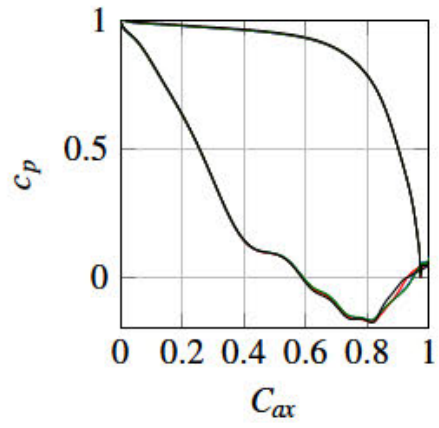

(a)

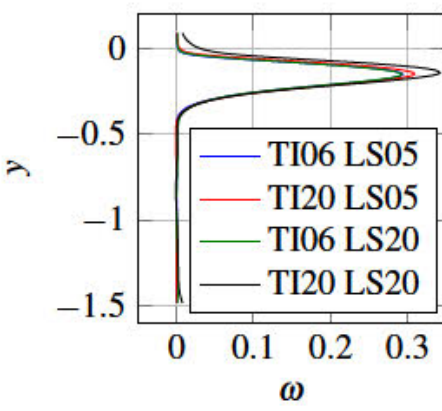

(b)
FIGURE 5: VARIATION OF PRESSURE DISTRIBUTION (A) AND KINETIC LOSS (B) FOR DIFFERENT INLET TURBULENCE STATES.

However, the blade then adopts quickly to the new inlet turbulence and comparing the interval starting at time 17.37 and spanning four time units, a significant shift of the transition upstream due to the presence of higher TI and greater LSs freestream turbulence can be observed. Crucially, when comparing the interval spanning the entire four time units to one spanning only the last two time units, starting at 19.44 , virtually the same result is obtained. These results imply that while the averaging time of approximately 20 eddy-rollover-times of the largest structures of the inlet turbulence does not appear to be sufficient to get fully converged statistics of the inlet turbulence, it is sufficient to obtain fully converged statistics of quantities on the blade. In addition to the wall normal heat flux, statistical convergence was also found for all other quantities reported in this paper but not shown for conciseness. It can also be concluded that the transient time the boundary layer requires to adapt to the new state of inlet turbulence seems to be approximately two time units. This quick adaptation to the new inlet turbulence is probably related to minor changes in the pressure distribution such that the potential flow field around the blade only requires minor adjustments in contrast to cases where earlier transition helps to suppress separation bubbles, as common for low pressure turbines.

\section{RESULTS} as

Figure 5 (a) presents the mean pressure distribution, defined

$$
c_{p}=\frac{p(x)-p_{2, m i x}}{p_{t 1, m i x}-p_{2, m i x}},
$$

where $p(x)$ is the pressure at the blade and $p_{t 1, \text { mix }}$ and $p_{2, \text { mix }}$ are the mixed out stagnation pressure at the inlet and the mixed out static pressure at the outlet, respectively. Despite minor differences between the various cases seen in the aft section on the suction side, mainly due to the effect of the different inlet turbulence states on suction side boundary layer transition, overall the global loading behavior is very robust. The kinematic wake loss, defined as

$$
\omega=\frac{p_{t 1, \text { mix }}-p_{t 2}(y)}{p_{t 1, \text { mix }}-p_{2, \text { mix }}}
$$

where the $p_{t 2}(y)$ is the stagnation pressure $10 \% C_{a x}$ downstream of the TE, is compared for the different cases in Figure 5 (b). As sug-

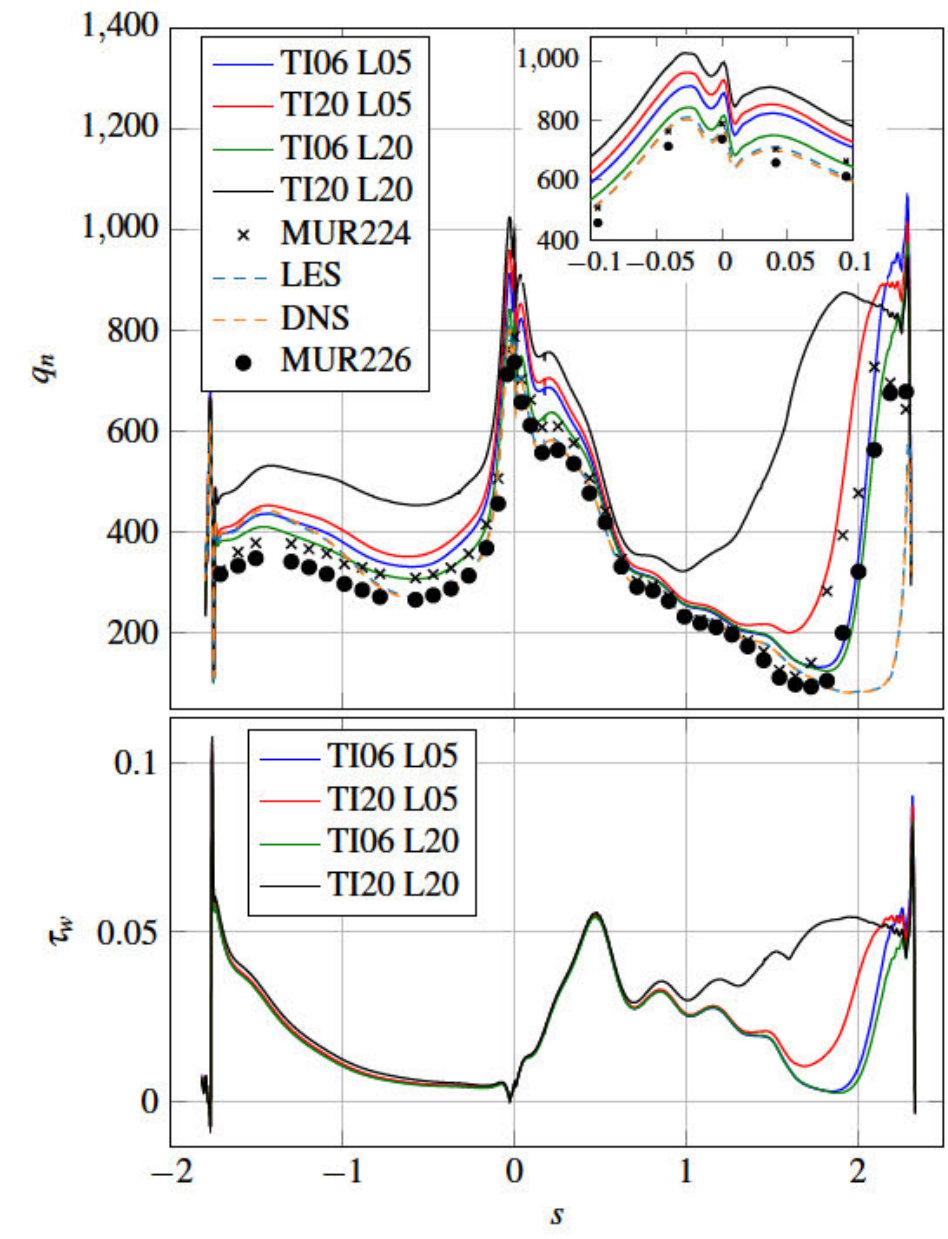

FIGURE 6: TOP: WALL HEAT FLUX ALONG THE BLADE SURFACE $S$ FOR THE FOUR SIMULATIONS CONDUCTED IN THIS STUDY, CASES MUR224 (6\% TURBULENCE AND LS $\approx 20 \%$ ) and MUR226 ( $4 \%$ TI AND LS $\approx 25 \%$ ) OF THE EXPERIMENT CAMPAING BY [2]. IN ADDITION AN LES PERFORMED ON THE SAME GRID AS USED IN THIS WORK IS COMPARED WITH THE DNS BY Wheeler et al. [12] (3.5\% TI and $L S \approx 2.5 \%$ ). BOTTOM: WALL SHEAR STRESS PLOTTED AGAINST THE SURFACE LENGTH.

gested by Mee [27], earlier transition can materialize in a change in the wake profile and the data from this study do indeed show slight differences of the wake profiles, mainly in the peak amplitude. While the differences between the two TI06 cases are negligible, the TI20 LS05 case shows a slightly higher peak value. For the TI20 LS20 cases the peak amplitude is even higher and also the wake width has changed, indicating more mixing or wider boundary layers.

\section{Mean wall heat flux and wall shear stress}

The heat flux to the wall is plotted in Figure 6 over the surface length where 0 is the leading edge. An insert in the upper right part shows a close up of the leading edge region. As discussed by Bhaskaran [28] and Wheeler et al. [12], the inlet turbulence structures reorient, are stretched and align with the blade surface such that their longest extent is in the streamwise direction. When convected close to the surface these structures induce higher heat flux across the surface, particularly at the leading edge and for 
the laminar pressure side. While this mechanism also is present on the suction side, it is superposed with another phenomenon, as discussed later.

At the leading edge (LE) the heat flux observed for the TI06 LS05 case is higher than for the TI06 LS20 case. This suggests that larger structures produce a smaller integral heat flux which could either be due to their smaller frequency or that large structures are not able to penetrate the BL as far as smaller structures. Note that for the TI06 cases it can be seen that the turbulence intensity of the TI06 LS20 case is closer to the experimental value in which an even larger LS was present. For the TI20 cases the TI at the leading edge is almost twice as high in the LS20 case compared to the LS05 case (see Figure 2). Conversely the LS of it is significantly larger such that overall the effect of TI dominates the influence of the larger LS and leads to a larger heat flux at the leading edge compared to the TI20 LS05 case.

For cases at the similar $R e_{i s}$ and $M a_{i s}$, Arts and Rouvroit [2] reported an increased heat transfer on the pressure side when changing TI from $1 \%$ to $3.5 \%$ and $6 \%$, where the difference between the latter two cases was smaller. With respect to the experiment, our case TI06 LS20 shows the closest agreement and in terms of LS and TI it is the case closest to the experimental inlet turbulence condition. Both cases that have a higher TI at the LE region (see Figure 2) show a higher heat flux, in accordance with the experiment. Conversely, the case TI06 LS05 has a slightly lower TI in the inlet region but it still exhibits a higher heat flux. Similar to the LE region, this is probably related to larger structures causing a smaller heat flux. With respect to the leading edge the quantitative differences between the cases TI06 LS05, TI06 LS20 and the TI20 LS05 are smaller. In contrast, the TI20 LS20 case shows large differences to the other three cases, and compared with the TI06 LS20 case the heat transfer is about $25 \%$ higher.

Changing TI from $1 \%$ to $3.5 \%$ and $6 \%$ on the suction side Arts and Rouvroit [2] found higher heat flux in the LE region. Farther downstream, the individual heat flux values got closer to each other until for each case transition occurred at different axial locations, where the earliest transition was observed for the highest TI. Similarly for the cases considered in this work the differences in the LE region progressively reduce when moving downstream on the suction side until all cases, except TI20 LS20, almost collapse at $s \approx 0.5$. This might be related to the strong acceleration region that is present up to approximately $s=1.3$ ( [3]). Both cases at TI06 remain similar for most of the suction side until transition starts at $s=1.95$ indicating that the influence of the LS in this area is small. The smaller LS case with TI06 has a slightly steeper growth of heat flux that starts slightly farther downstream. However, both cases are fairly similar. The TI20 LS05 case has slightly higher heat flux levels in the range $0.75<s<1.6$ but follows a similar trend with respect to the TI06 cases. A sharp rise at about $s=1.8$ is observed, indicating the onset of transition and the subsequent growth rate is slightly smaller compared to the two TI06 cases. The earliest onset of transition is present in the TI20 LS20 case. While after the leading edge the heat flux reduces more rapidly with respect to the other cases and follows a similar trend at $s \approx 1$, it starts increasing again and rises sharper at $s=1.4$ which could be considered as the mean onset of transition. With respect to the other cases the growth rate of heat flux in this transition region is more benign.

Hence, the simulations presented in this work are in qualitative agreement with what is expected based on previous work. However, when quantitatively comparing case TI06 LS20 to the experimental data from case MUR224 which is at similar condi- tions in terms of operating point and inlet turbulence, the transition onset in the simulations appears to be delayed. While the LES setup has been tailored to resemble the experiment there are a number of differences, including the inlet turbulence state and other fluctuations in the inlet turbulence spectra, wall roughness, endwall blockage, filtered blade coordinates [12] and slightly different operating conditions, each of them with the potential to affect transition. While some of the differences could be tackled by additional simulations, others like the additional peak in the inlet spectra, wall roughness and endwall blockage cannot be ruled out with the current setup and including those would substantially increase the cost of the simulation while others will remain uncertainties. Since a quantitative agreement is difficult given the uncertainties with regards to the exact experimental setup, we want to emphasize that all numerical quality checks including domain size independence and grid convergence have been conducted such that the LES setup is reliable. To further verify the quality of the LES setup used, an LES performed with the identical setup as in the DNS by Wheeler et al. [12], except for a significantly coarser grid, was performed. Figure 6 shows that the LES and the DNS data are almost identical. Thus differences observed for the other cases reported here are due to the differences of the setup only.

The agreement with experimental data [2] in terms of transition location is best for the TI20 LS05 case. However, the distribution of $q_{n}$ in the region $1.5 \lesssim s \lesssim 1.8$ differs, with the curent data bypassing the dip in heat flux seen in the experiments before the substantial rise. Also, in this case the inlet TI is substantially higher. While both TI06 cases have the same TI at the inlet as in the experiment, they show later transition. Considering the difference of the LS in this work to the experiment the trends that occur when changing the LS might help to understand the difference to the experiment. It can be seen that the larger LS shows better agreement with experiments for most of the vane, except for the transition onset, which is delayed. Indeed, the transition onset is slightly farther upstream for the case with smaller length scales. Scrutinizing the spectrum presented in [2], it is interesting to note that the turbulence does not only show a $-5 / 3$ decay behavior, typical of turbulence, but also a peak at higher frequency that can be related to the vortex shedding frequency of the bars in the inlet region, as discussed by Jee et al. [23].

Another quantity that is helpful for investigating transition is the wall shear stress, $\tau_{w}$, presented in Figure 6 (bottom). In contrast to the heat flux, the wall shear stress is similar in the LE region for all cases and only the TI20 LS20 case shows slightly higher levels on the pressure side. As discussed above $q_{n}$ rises for higher TI because of streaks wrapping around LE, however, that does not affect mean $\tau_{w}$. The main differences can be observed on the suction side from about $s=0.75$ onwards for the case TI20 LS20, while all other cases remain similar until $s=1.3$. Again the two TI06 cases show relatively similar transition behavior. Particularly in the TI20 LS20 the increase in wall shear stress is more continuous than the increase in heat flux and it is less clear where transition starts. It should also be noted that none of the cases performed show negative wall shear stress on the suction side, i.e. no separation bubble is present, in contrast to the DNS case of Wheeler et al. [12] in which a small separation bubble was observed. However, there the inlet TI was roughly $3.5 \%$ and had decayed to even smaller values when reaching the blade. While the current TI06 cases appear to be on the verge of separation in the mean just upstream of $s=2$, and thus might exhibit instantaneous flow separation, as will be discussed later, the TI20 cases are far from featuring separated 
flow.

\section{Instantaneous Flow Features}

To get a better understanding of the mechanisms leading to the changes in heat flux and transition, snapshots of iso-surfaces of the complex part of the eigenvalue of the velocity gradient tensor at a value of $\lambda_{c i}=200$ are presented in Figure 7 for different cases. The iso-surfaces are colored by the spanwise velocity. Using a high value of $\lambda_{c i}$ allows to visualize structures only in highly turbulent regions like the turbulent boundary layers and turbulent spots since those are characterised by higher rotation rates compared to the free stream flow. Some structures in the free stream can be seen but do not block the view onto the turbulent structures at wall.

In Figure 7 (a) and (b) two randomly selected snapshots of the case TI06 LS05 are presented. For both instances the transition onset is limited to the final $10 \%$ of the blade surface and there are only a few other snapshots over time that show structures further upstream. In general there are several spanwise locations where transition starts at a similar axial location (Figure 7 (a)). However, for some instances the BL does not transition at all for a part of the span (Figure 7 (b)) and the laminar boundary layer extends to the TE. As might be expected from the heat flux plots, snapshots for the TI06 LS20 cases show a similar behaviour ad are therefore not shown here for conciseness. The major difference between the TI06 LS20 and the TI06 LS05 cases is that the turbulent spots are wider spread in the spanwise direction which might explain the slightly later transition onset in the mean heat flux. Even though the spanwise oriented rollers reported by Wheeler et al. [12] for this case are not seen in Figure 7 they are present upstream of the turbulent regions shown. These spanwise coherent structures have also been identified to be present in the TI20 LS05 case.

For the TI20 LS05 case the first transitional structures are present further upstream than in the TI06 cases, as can be seen in Figure 7 (c) where a spot (indicated with letter A) is present already at about $40 \%$ of the surface length. Further downstream the turbulent BL almost covers the entire span. In the subsequent snapshot this aft turbulent region has been washed out and followed by a laminar region, reminiscent of what has been referred to as a calmed region [6], even though further investigations are required to verify that a comparison with unsteady cases is valid. The small upstream turbulent region seen in Figure 7 (c) grows both in the span- and streamwise directions until it merges with the turbulence in the downstream region as shown in Figure 7 (d) (indicated with letter A). However, at the right-hand part of the domain no turbulent spot was present and the laminar region almost extends to the TE. In Figure 7 (d) two more spots (indicated with letters B and C) can be seen at different axial positions that subsequently develop into turbulence as well. With respect to the turbulent spots in the TI06 LS05 case these spots occur earlier, but seem to be less frequent. Since they are sparser, they grow wider in the span and longer in streamwise directions, before they merge with the surrounding spots or the turbulent boundary layer further downstream.

In Figure 7 (e) and (f) two instances for case TI20 LS20 are presented. With respect to the TI20 LS05 case it has both a higher TI and a higher LS when measuring at the LE. As expected the first spots occur even further upstream and again grow and eventually lead to transition. The time difference between the two snapshots is such that the small spot on the right of the domain (indicated with letter $\mathbf{D}$ in Figure 7 e) develops into the large spot about half way down the blade that starts catching up with the downstream turbulent area, seen in Figure 7 (f) (letter D). While it is known that streaks grow in the streamwise direction more than in the spanwise direction [10], the locations where spots appear seem to be proceeded by further spots as can be seen in Figure 7 (e) in the spanwise region indicated by arrow. The same region shows turbulent spots as well at a later time in Figure 7 (f) while there are no turbulent spots for other spanwise locations in either Figure (e) or (f). From theory it is expected that a turbulent spots is succeeded by a calmed region and therefore less receptive to further spots. This can be explained by considering the stretching of turbulent structures in the streamwise direction that has previously been discussed by Wu and Durbin [29] for the pressure side boundary layer of low pressure turbines. Similar to their scenario the vortices of the inlet turbulence are reoriented in the streamwise direction and are stretched by the velocity field such that the they span almost the entire suction side length. While this mechanism is present in the cases discussed above, it is less pronounced for two reasons. Firstly, for the small LS cases the structures do not reach the same lengths and are probably convected by faster, such that the number of turbulent spots induced by the same vortex is smaller. Secondly, the higher TI leads to earlier onset of transition such that this phenomenon is easier to observe since it can occur over a longer surface length.

\section{Histograms of wall shear stress}

In all cases shown in Figure 7 a time intermittent change between laminar and turbulent regimes can be seen that is more pronounced for higher TI and higher LS. Hence, characterising the heat flux and wall shear stress by considering only mean quantities might not be sufficient. To get an impression of the likelihood of transition occurring at a certain location, normalized histograms are presented in Figure 8 for the four cases considered in this work. At each streamwise location along the blade a histogram of the wall shear stress evaluated over the spanwise direction and time (200 snapshots separated by 0.01 time unites) is computed and the histogram at each streamwise location is normalized such the integral over the range is unity. A higher maximum value and a thinner band indicates less variation in wall shear stress at a particular streamwise location. For the TI06 cases the band of probable realizations is relatively small up to $\mathrm{x}=0.9$. It can be seen that both TI06 cases have instances where a separation occurs even though the mean flow didn't show a separation bubble. Close to the TE the spread in both cases significantly increases, with the spread starting to increase slightly earlier for TI06 LS20 case.

For the TI20 LS05 case the band of probable realizations is wider already from the LE. However, it seems to narrow down downstream of $x=0.4$ until the spread signficantly increases again from $x=0.8$. While the center of the band with most realizations shows a similar trend compared to the TI06 cases in the interval $0.8 \leq x \leq 0.9$ the profile is skewed towards higher values of wall shear stress, representing the instances where the transition onset is earlier. Also the TI20 LS05 case has temporal or spanwise instances where the flow separates, however, transition occurs earlier as discussed above.

The TI20 LS20 case shows the widest band of realizations of wall shear stress. Since both the TI and the LS are larger it is not clear if it is an effect of the either TI or LS, or the combined action. With respect to the TI06 cases the spread of the band is about twice as wide and slightly skewed towards larger wall shear stress events 

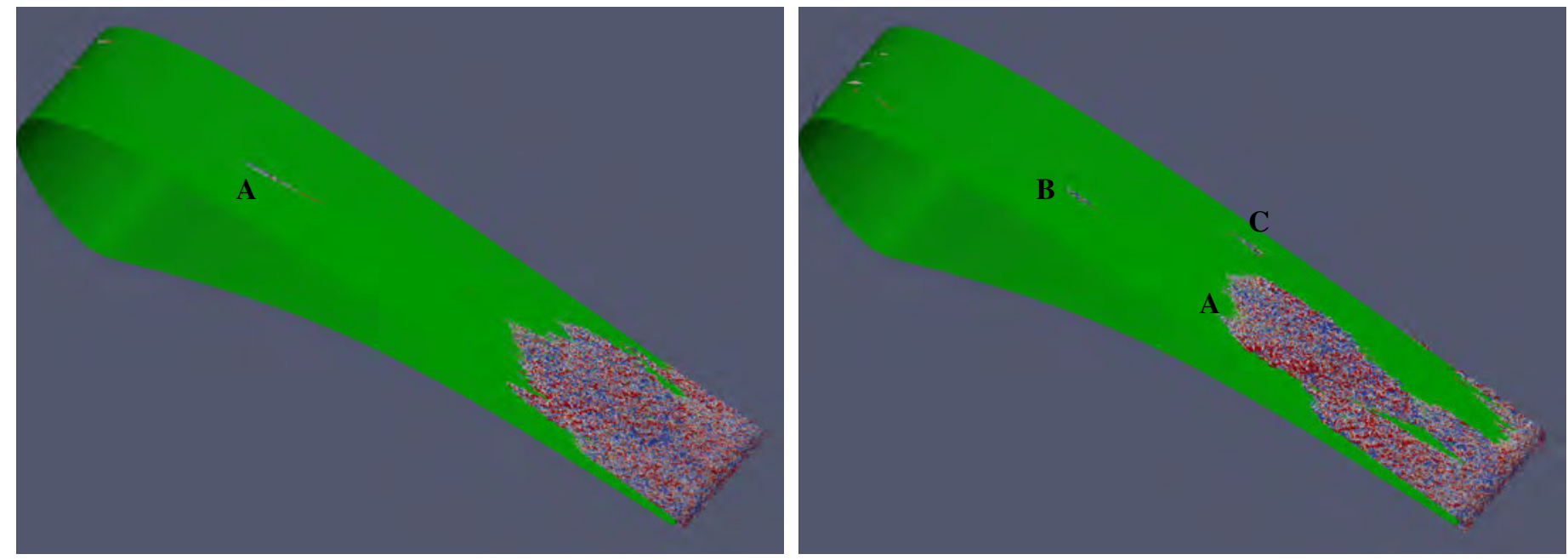

(c)

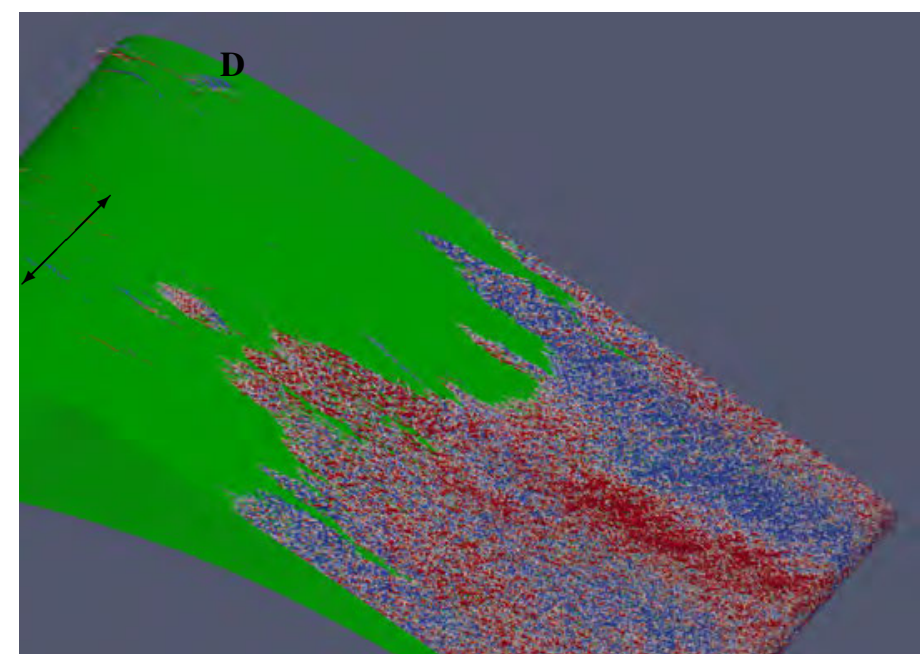

(e) (d)

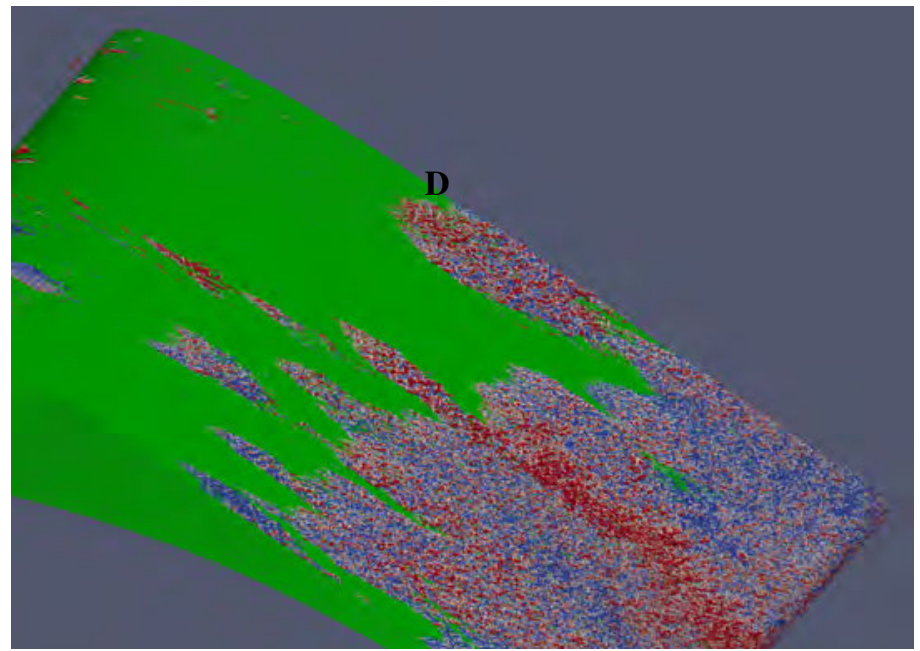

(f)

FIGURE 7: TWO SNAPSHOTS IN TIME OF THE FLOW ON THE SUCTION SIDE FOR THREE CASES: FIRST ROW TI06 LS05; SECOND ROW TI20 LS05 AND THIRD ROW TI20 LS20. THE STRUCTURES ARE VISUALIZED USING ISOSURFACES OF THE COMPLEX PART OF THE EIGENVALUE OF THE VELOCITY GRADIENT TENSOR AT A VALUE OF $\lambda_{C I}=200$. ARE COLORED USING SPANWISE VELOCITY $W$ IN THE INTERVAL OF -0.1 (BLUE) TO 0.1 (RED). 


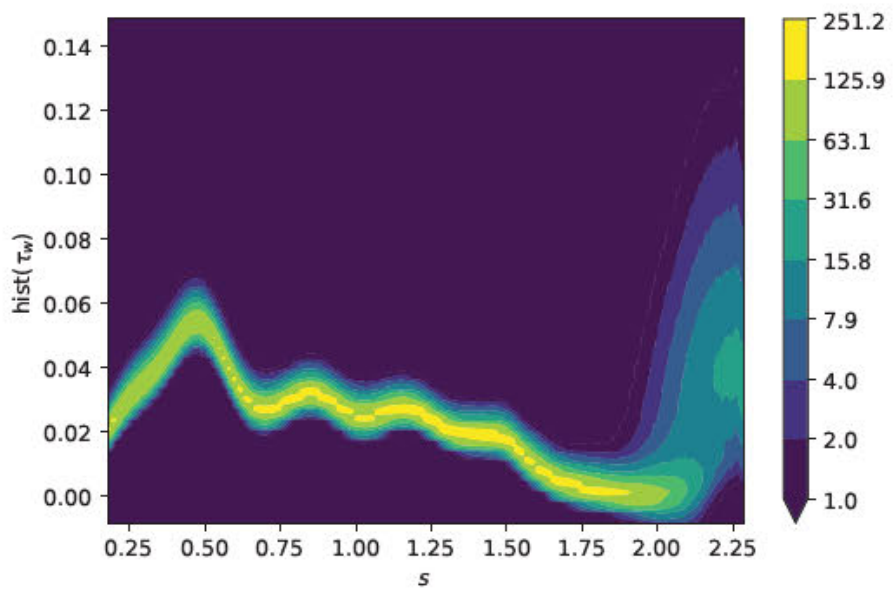

(a) TI06 LS05

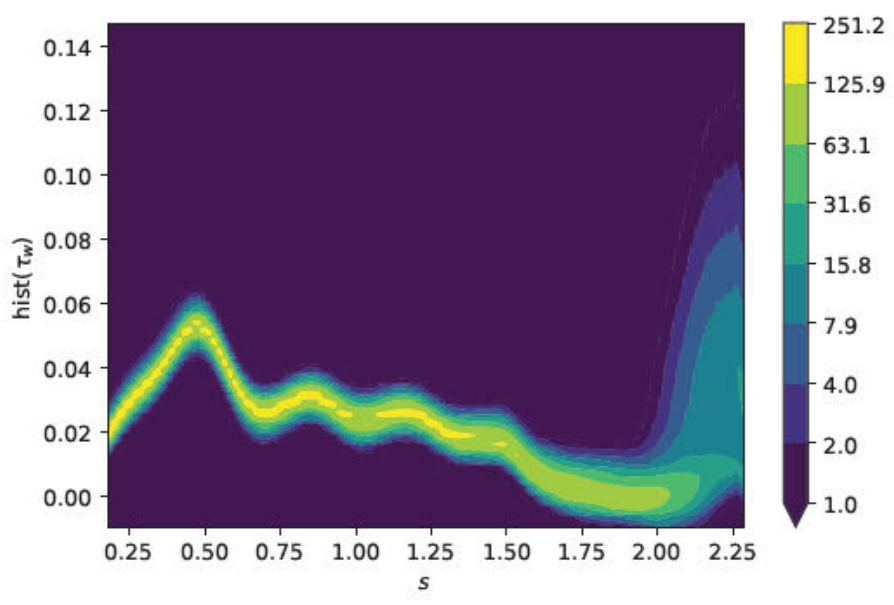

(c) TI06 LS20

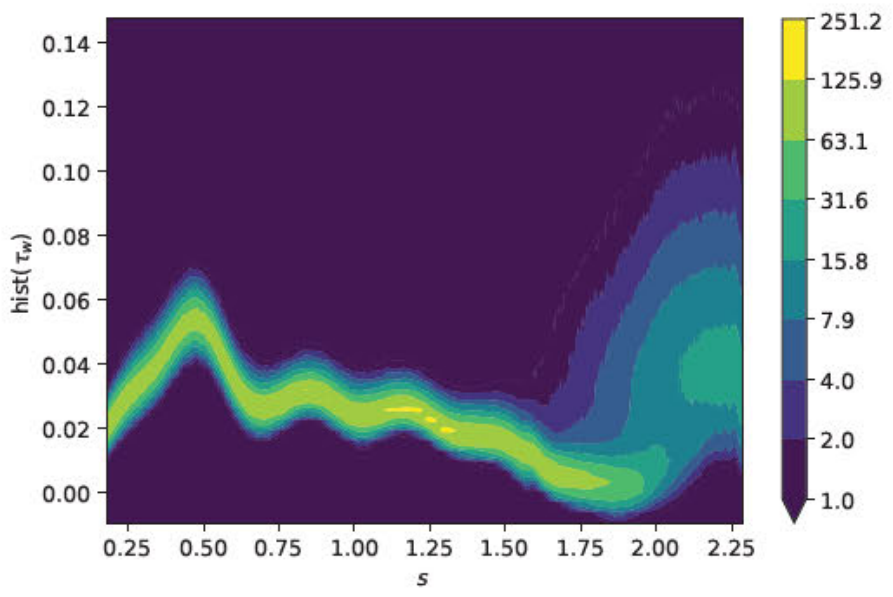

(b) TI20 LS05

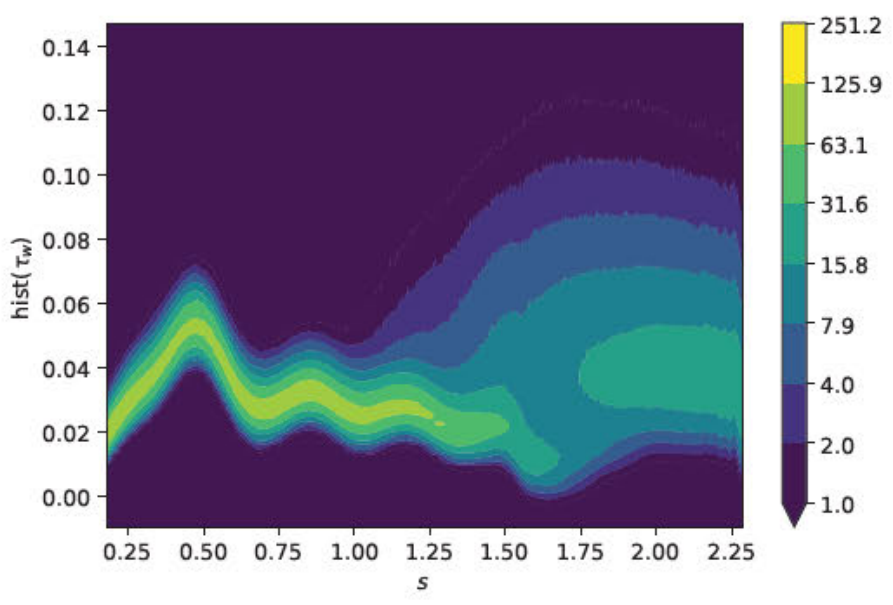

(d) TI20 LS20

FIGURE 8: HISTOGRAMS OF WALL SHEAR STRESS ON THE SUCTION SIDE FOR THE CASES PRESENTED IN THIS WORK.

that are related to the turbulent spots developing earlier in this case. Starting at $x \approx 0.65$ the spread starts increasing towards larger wall shear stresses while the core of the band is only slightly higher than for the other case. This reflects the interchange of laminar with turbulent phases. However, at $x \approx 0.8$ the core disappears and while there are still low wall shear stress events occurring downstream of 0.8 those are less frequent. Indeed there are realizations where the laminar regions extend to the trailing edge, while first turbulence structures can be seen already at $s=0.5$ (see Figure 7(f)).

\section{Wall normal velocity profiles}

The influence of the inlet turbulence on the boundary layer profiles is visualized in Figure 9 for different locations along the suction side surface. At each location time averaged profiles of velocity tangential to the blade as well as kinetic energy of the fluctuating field are presented, where $d$ is the wall normal distance. The tangential velocity is While $k$, defined as

$$
k=\frac{\tau_{11}+\tau_{22}+\tau_{33}}{\bar{\rho}},
$$

is generally referred to as turbulence kinetic energy, in case of intermittent laminar and turbulent BL profiles, a larger part of the variation might be related to the changing shape of the mean flow profile in time, such that it is not turbulence kinetic energy in the classic sense. Close to the leading edge at $\mathrm{s}=0.07$ (Figure 9 (a)) the tangential velocity profiles are fairly similar with only minor variations close to the boundary layer thickness. These are probably related to differences in turbulent mixing due to the differences in $k$. In the freestream all profiles of $k$ approach the freestream value and it can be seen that there is a direct relation between $k$ in the freestream and the leading edge. All profiles subsequently develop a peak in the outer region of the boundary layer (Figure 9(b)) as reported by Radomsky and Thoule [30]. The peaks are more pronounced for small LS which have a larger rise with respect to the freestream value. Brandt et al. [10] reported that turbulence with the same intensity is able to penetrate deeper in to a boundary layer if the LS is smaller, which is in accordance with the current observations. However, further investigations are required to understand if this is indeed the mechanism responsible for the peaks seen in the $k$ profiles. Up to $s=0.7$ (Figure 9 (c)) the amplitude for all cases grow, where the growth is greater for the large LS cases. For all cases the peaks in $k$ are at least a factor of 2 larger than the respective free stream value while their distance to the wall is the 


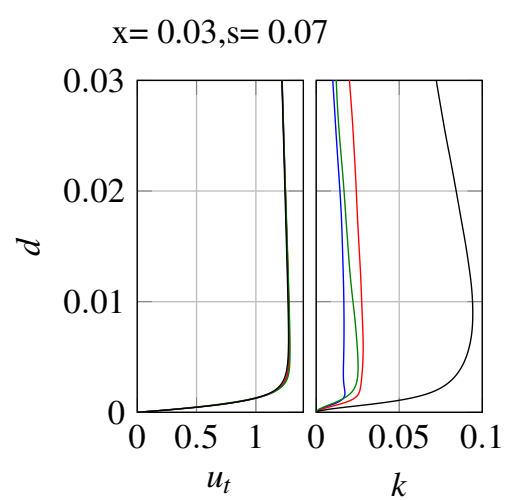

(a)

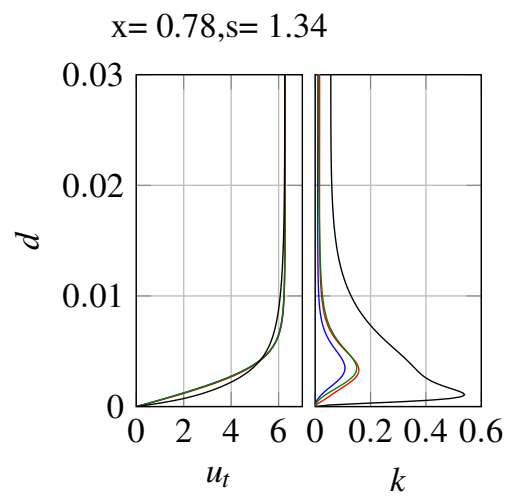

(d)

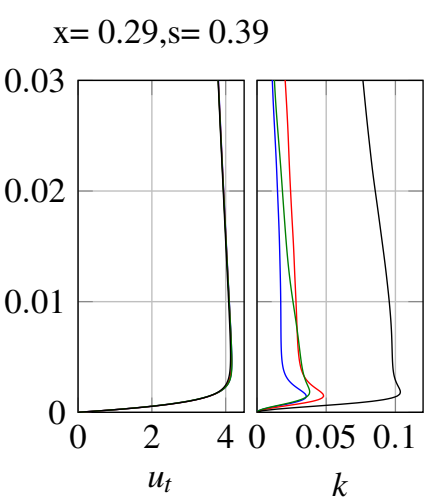

(b)

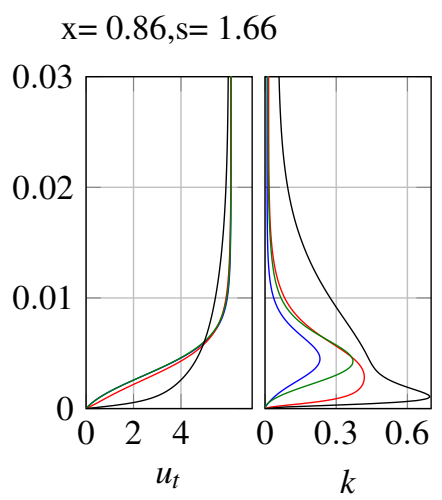

(e)

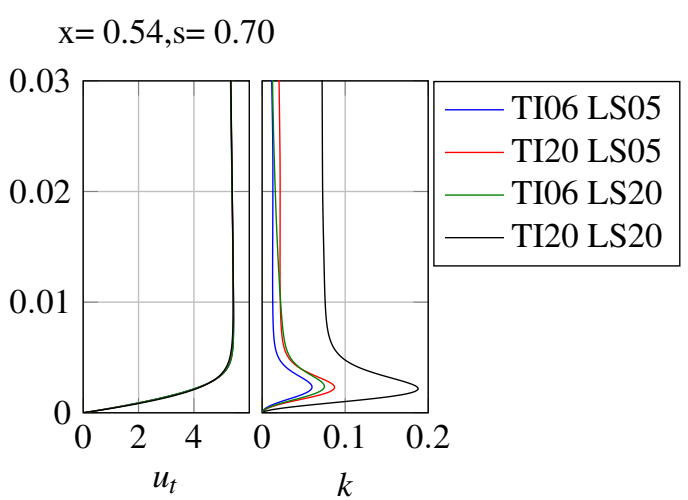

(c)

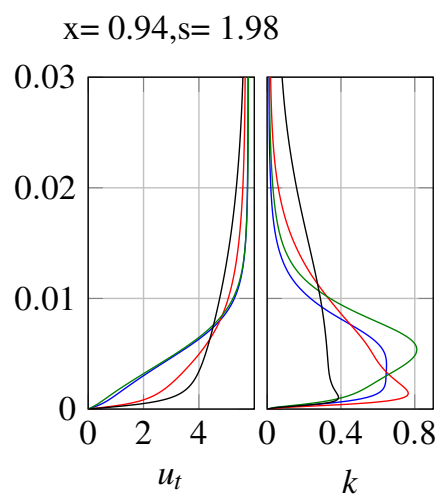

(f)

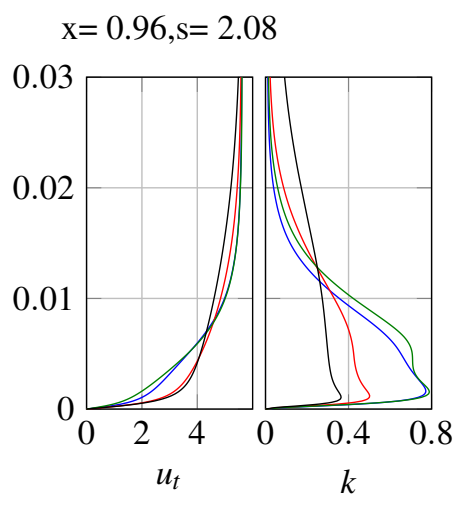

(g)

FIGURE 9: WALL NORMAL PROFILES OF TANGENTIAL VELOCITY AS WELL AS TURBULENCE KINETIC ENERGY FOR DIFFERENT POSITION ALONG THE SURFACE LENGTH.

same. The tangential velocity profiles are virtually the same for all cases up to $s=0.7$ and thus classical linear instability growth would be expected to be the same for all cases up to this point.

Up to $s=1.34$ (Figure 9 (d)) the amplitudes for all $k$ profiles grow, where the growth in the TI06 LS20 case is larger than in the TI06 LS05 and TI20 LS05 cases. Indeed at $s=1.34$ the TI06 LS20 case has almost caught up with the TI20 LS05 case. Again referring to the work on flat plates by Brandt et al. [10], it is known that in their scenario a larger LS resulted in faster growth, but it yet has to be verified if the same mechanisms is acting here as well. In the wall normal direction the peaks of TI06 LS20, TI06 LS05 and TI20 LS05 are at the same wall normal distance and with respect to $s=0.7$ the wall normal distance has increased from about 0.0025 to 0.004 . At $s=1.34$ the TI20 LS20 case has developed a near wall peak that is substantially closer to the wall $(d=0.0015)$ than what is seen in any of the other cases and is at a position where the near wall peak of a turbulent BL is expected. Considering the wall heat flux at this position and the tangential wall profile, it appears that the boundary layer is not fully turbulent yet, but shows transitional character. The scenario by which the peak in $k$ transforms to a different shape is the same in all cases (and similar to the flate plate plate work by Brandt et al. [10]) and is shown in Figures 9 (e)-(g) for case TI06 LS20. At $s=1.66$ (Figure 9 (e)) the $k$ profile of TI06 LS20 has a single peak at approximately $d=0.004$. Subsequently an inner peak develops at $d=0.0015$ that can be seen in Figure 9 (f)). Up to this stage the outer peak increases in magnitude. At $\mathrm{s}=2.08$ (Figure $9(\mathrm{~g})$ ) the inner peak dominates and the outer peak already decayed with respect to $s=1.98$. As can be seen for case
TI20 LS20 in Figures 9 (d)-(g), the inner peak first increases before substantially decreasing in amplitude. In a turbulent boundary layer near wall turbulence leads to an increase in mixing such that the mean profile of a turbulent $\mathrm{BL}$ is fuller with respect to a laminar BL. This difference in the changing mean profiles leads to an increased $k$. Hence, the high values of $k$ are considered to be partly related to near wall turbulence and to the variation in the mean flow profile. When approaching a fully turbulent state the contribution to $k$ by the intermittent mean profile will reduce. This is also reflected in the heat flux (Figure 6). The start of the slope indicating transition in the heat flux corresponds with the appearance of the inner peak for all cases. Then $k$ increases rapidly and the peak value is found roughly at the axial location where half the rise in $q_{n}$ towards the fully turbulent regime has occurred (e.g. at $s \approx 1.6$ for TI20 LS20). Once the turbulent regime is reached, the $k$ values stabilize, see for example Figures 9 (f)-(g) for the TI20 LS20 case.

While transition appears to occur following the same steps for all cases, suggesting that the underlying mechanisms are the similar, the location $s$ at which the process starts is different for each case. The axial positions at which the cases develop the inner peak are the same at which they transition. However, the appearance of the inner peak is not related to the magnitude of $k$ in the boundary layer. In particular, while the magnitude of the peak in $k$ for the TI06 LS20 case is almost twice as high as for the TI06 LS05 case, the latter transitions earlier. Summarizing the development of the TI06 cases it can be stated that both have a similar free stream level of $k(d>0.03)$ at all positions along the surface. Close to the leading edge the LS05 case first develops a peak but the growth of the 
peak in the LS20 case is larger. Still the LS05 case develops the inner peak earlier and transitions faster.

\section{Conclusions}

A series of highly resolved LES, using a in-house highly accurate compressible Navier-Stokes solver, of an HPT vane at $R e_{i s}=540,000$ and $M_{i s}=0.92$ were conducted, varying the inlet turbulence conditions both in terms of the turbulence intensity and the turbulence length scales. Particular care was taken to ensure that the domain size was sufficiently large to capture the largest imposed length scales, $20 \%$ of the axial chord. This was achieved by performing a precursor study of the turbulent inlet region only. The largest turbulence intensity investigated for the full vane configuration was $20 \%$. The analysis focused on characterizing the inlet turbulence and quantifying the effect of the inlet turbulence variations on the vane boundary layers, in particular the heat flux to the blade. From spectra of the inlet region it was found that given a fixed turbulence intensity TI, smaller imposed length scales LS resulted in more energy in the smaller scales, i.e. a shift of the peak in the spectrum towards higher frequencies. In the case with TI $20 \%$ and $5 \%$ length scale, the rapid axial decay of the inflow turbulence resulted in a turbulence level at the leading edge close to that of the case with TI $06 \%$ and $20 \%$ length scale, albeit with a significantly larger length scale. This larger length scale resulted in smaller values of turbulence kinetic energy at the leading edge, with faster growth of the amplitude of $k$ moving downstream. The heat flux of the case with larger length scales was also smaller, suggesting smaller length scales penetrating deeper into the boundary layer and producing perturbations leading to increased levels of $q_{n}$. Overall, the case with TI $20 \%$ and 5\% length scale exhibited earlier transition at the aft section of the suction side than the cases with smaller TI. In the case with TI $20 \%$ and $20 \%$ length scale, the inlet turbulence maintained high levels all the way to the leading edge, i.e. exhibited only marginal decay, and the heat flux levels were much greater than for all other cases over the entire blade. In particular, transition to turbulence occurred much earlier. Snapshots of the flow at different instances in time, and probability functions of the wall shear stress, have elucidated the highly intermittent and local nature of transition, with the flow in some instances remaining laminar up to the trailing edge, and the transition location moving up and down considerably. By studying the evolution of the boundary layer velocity and turbulence kinetic energy profiles over the suction surface initial suggestions on possible transition scenarios have been made.

\section{ACKNOWLEDGEMENTS}

This research used resources of the Oak Ridge Leadership Computing Facility at the Oak Ridge National Laboratory, which is supported by the Office of Science of the U.S. Department of Energy under Contract No. DE-AC05-00OR22725. Further, this work was supported by a grant from the Swiss National Supercomputing Centre (CSCS) under project ID S622.

\section{REFERENCES}

[1] Leach, K. P., 1983. Energy Efficient Engine High Pressure Turbine Component Rig Performance Test Report NASA PWA-559-243. Tech. rep.
[2] Arts, T., Lambert de Rouvroit, M., Rutherford, A., 1990. "Aero-thermal investigation of a highly loaded transonic linear turbine guide vane cascade. A test case for inviscid and viscous flow computations". In Proc. of the ASME IGTI 90GT-358.

[3] Nix, A. C., 2003. "Effects of High Intensity, Large-Scale Freestream Combustor Turbulence on Heat Transfer in Transonic Turbine Blades". PhD thesis.

[4] Laskowski, G., Ledezma, D., Tolpadi, A., and Ostrowski, M., 2008. "CFD simulations and conjugate heat transfer analysis of a high pressure turbine vane utilizing different cooling configurations". In ISROMAC-12.

[5] Dees, J. E., Bogard, D. G., Ledezma, G. a., and Laskowski, G. M., 2013. "Overall and Adiabatic Effectiveness Values on a Scaled Up, Simulated Gas Turbine Vane”. Journal of Turbomachinery, 135(5), p. 051017.

[6] Coull, J. D., and Hodson, H. P., 2011. "Unsteady boundarylayer transition in low-pressure turbines". Journal of Fluid Mechanics, 681(1), jul, pp. 370-410.

[7] Klebanoff, P., Tidstrom, K., and Sargent, L., 1962. "The three-dimensional nature of boundary-layer instability". $J$. Fluid Mech.

[8] Durbin, P., and Wu, X., 2007. "Transition Beneath Vortical Disturbances". Annual Review of Fluid Mechanics, 39, pp. 107-128.

[9] Westin, K. J. a., Boiko, a. V., Klingmann, B. G. B., Kozlov, V. V., and Alfredsson, P. H., 1994. "Experiments in a boundary layer subjected to free stream turbulence. Part 1. Boundary layer structure and receptivity". Journal of Fluid Mechanics, 281(1994), p. 193.

[10] Brandt, L., Schlatter, P., and Henningson, D. S., 2004. "Transition in boundary layers subject to free-stream turbulence". Journal of Fluid Mechanics, 517, pp. 167-198.

[11] Mayle, R., 1991. "The Role of Laminar-Turbulent Transition in Gas Turbine Engines". ASME J. Turbomach., 113, pp. 509-536.

[12] Wheeler, A., Sandberg, R., Sandham, N., Pichler, R., Michelassi, V., and Laskowski, G., 2016. "Direct numerical simulations of a high-pressure turbine vane". Journal of Turbomachinery, 138(7).

[13] Pichler, R., Kopriva, J., Laskowski, G., Michelassi, V., and Sandberg, R., 2016. "Highly Resolved Les of a Linear Hpt Vane Cascade Using Structured and Unstructured Codes". Turbomachinery Technical Conference and Exposition GT2016, pp. 1-10.

[14] Sandberg, R. D., Michelassi, V., Pichler, R., Chen, L., and Johnstone, R., 2015. "Compressible Direct Numerical Simulation of Low-Pressure Turbines -Part I: Methodology". ASME J. Turbomach., 137(5), p. 051011.

[15] Michelassi, V., Chen, L.-w., Pichler, R., and Sandberg, R. D., 2015. "Compressible Direct Numerical Simulation of LowPressure Turbines Part II : Effect of Inflow Disturbances". Journal of Turbomachinery, 137(July), pp. 1-12.

[16] Carpenter, M. H., Nordström, J., and Gottlieb, D., 1999. "A Stable and Conservative Interface Treatment of Arbitrary Spatial Accuracy”. J. Comp. Phys., 148(2), jan, pp. 341-365.

[17] Wheeler, A. P. S., and Sandberg, R. D., 2013. "Direct Numerical Simulations of a Transonic Tip Flow with Free-stream Disturbances". In ASME Turbine Blade Tip Symposium TBTS2013-2037.

[18] Nicoud, F., and Ducros, F., 1999. "Subgrid-scale stress mod- 
elling based on the square of the velocity gradient tensor". Flow, Turbulence and Combustion, 62(3), pp. 183-200.

[19] Michelassi, V., Chen, L., Pichler, R., Sandberg, R., and Bhaskaran, R., 2016. "High-Fidelity Simulations of LowPressure Turbines: Effect of Flow Coefficient and Reduced Frequency on Losses". Journal of Turbomachinery, 138(November), pp. 1-12.

[20] Bogey, C., de Cacqueray, N., and Bailly, C., 2009. "A shockcapturing methodology based on adaptative spatial filtering for high-order non-linear computations". J. Comp. Phys., 228(5), pp. 1447-1465.

[21] Sandberg, R. D., Jones, L. E., and Sandham, N. D., 2006. “A zonal characteristic boundary condition for numerical simulations of aerodynamic sound". In European Conference on Computational Fluid Dynamics, ECCOMAS CFD 2006, P. Wesseling, E. Oñate, and J. Périaux, eds.

[22] Fontaneto, F., 2014. "Aero-Thermal Performance of a FilmCooled High Pressure Turbine Blade/Vane: A Test case for Numerical Codes Validation". PhD thesis, Universita degli studi di Bergamo.

[23] Jee, S., Joo, J., and Medic, G., 2016. "Large-Eddy Simulation of a High-Pressure Turbine Vane with Inlet Turbulence". Turbomachinery Technical Conference and Exposition GT2016, pp. 1-12.

[24] Klein, M., Sadiki, A., and Janicka, J., 2003. "A digital filter based generation of inflow data for spatially developing direct numerical or large eddy simulations". J. Comp. Phys., 186(2), pp. 652-665.

[25] Xie, Z. T., and Castro, I. P., 2008. "Efficient generation of inflow conditions for large-eddy simulation of streetscale flows". Flow, Turbulence and Combustion, 81(3), pp. 449-470.

[26] Touber, E., and Sandham, N. D., 2009. "Large-eddy simulation of low-frequency unsteadiness in a turbulent shockinduced separation bubble”. Theor. Comp. Fluid Dyn., 23(2), pp. 79-107.

[27] Mee, D. J., Baines, N. C., Oldfield, M. G. L., and Dickens, T. E., 1992. "An examination of the contributions to loss on a transonic turbine blade in cascade.". Journal of Turbomachinery, 114(January 1992), pp. 155-162.

[28] Bhaskaran, R., and Lele, S. K., 2010. "Large eddy simulation of free-stream turbulence effects on heat transfer to a highpressure turbine cascade". Journal of Turbulence, 11(February 2014), p. N6.

[29] Wu, X., and Durbin, P. A., 2001. "Evidence of longitudinal vortices evolved from distorted wakes in a turbine passage". Journal of Fluid Mechanics, 446, pp. 199-228.

[30] Radomsky, R. W., and Thole, K. a., 2002. "Detailed Boundary Layer Measurements on a Turbine Stator Vane at Elevated Freestream Turbulence Levels". Journal of Turbomachinery, 124(1), p. 107. 


\section{University Library}

\section{- M M N E R VA A gateway to Melbourne's research publications}

Minerva Access is the Institutional Repository of The University of Melbourne

Author/s:

Pichler, R;Sandberg, RD;Laskowski, G;Michelassi, V

Title:

HIGH-FIDELITY SIMULATIONS OF A LINEAR HPT VANE CASCADE SUBJECT TO VARYING INLET TURBULENCE

Date:

2017-01-01

Citation:

Pichler, R., Sandberg, R. D., Laskowski, G. \& Michelassi, V. (2017). HIGH-FIDELITY SIMULATIONS OF A LINEAR HPT VANE CASCADE SUBJECT TO VARYING INLET TURBULENCE. PROCEEDINGS OF THE ASME TURBO EXPO: TURBINE TECHNICAL CONFERENCE AND EXPOSITION, 2017, VOL 2A, 2A-2017, AMER SOC MECHANICAL ENGINEERS. https://doi.org/10.1115/GT2017-63079.

Persistent Link:

http://hdl.handle.net/11343/241900 